Pacific Journal of Mathematics

RELATIVE SATELLITES AND DERIVED FUNCTORS OF 


\title{
RELATIVE SATELLITES AND DERIVED FUNCTORS OF FUNCTORS WITH ADDITIVE DOMAIN
}

\author{
ULRICH OBERST
}

\begin{abstract}
This paper deals with relative satellites and derived functors of functors from an additive category $\mathfrak{U}$ into an Abelian category. The satellites and derived functors are defined by universal properties relative to classes $\subseteq$ of morphisms of $\mathfrak{U}$ that contain all morphisms whose domain is an initial object of $\mathfrak{Y}$, that are closed under multiplication and basecoextension, and whose elements have cokernels. The existence of satellites and derived functors relative to $\subseteq$ is shown by a method due to $D$. Buchsbaum without using the existence of either enough $\mathfrak{S}$-injective or $\mathfrak{S}$-projective objects in $\mathfrak{A}$. With the proper notion of $\widetilde{S}$-exactness in $\mathfrak{A}$ the exactness of the long satellite resp. derived functor sequence is established under quite general assumptions.
\end{abstract}

In the absolute case where $\mathfrak{A}$ is an Abelian category and $\mathfrak{S}$ is the class of all monomorphisms of $\mathfrak{A}$ the satellites resp. derived functors relative to $\subseteq$ are the well-known absolute satellites resp. derived functors as defined by H. Cartan and S. Eilenberg [2, Ch. 3 and 5] resp. P. Gabriel [4, Ch. 2]. The existence and exactness theorems $4.3,4.5,4.6,4.7,4.9,5.2,5.5$ and 5.6 of this paper especially furnish the corresponding theorems of H. Cartan and S. Eilenberg [2, Ch. 3 and 5], A. Grothendieck [5, Ch. 2], D. Buchsbaum [1], P. Gabriel [4, Ch. 2], and H. Röhrl [9]. ${ }^{1}$

If $\mathfrak{A}$ is Abelian and $\mathfrak{S}$ is the class of morphisms of an injective structure on $\mathfrak{A}$ in the sense of J. Maranda [7] then one recovers the results of [8]. In the preceding example the assumption that $\mathfrak{A}$ is Abelian is not necessary as has been shown by S. Eilenberg and J. C. Moore [3].

The results of this paper apply in particular to the following cases $(\S 6)$

( I ) The category $\mathfrak{A}$ has enough $T$-S-injective objects. In the absolute case one obtains an improvement of the results of $\mathrm{H}$. Röhrl [9].

(II) For every object $A$ in $\mathfrak{X}$ the preordered class $\mathfrak{S}(A)$ of all morphisms in $\subseteq$ with domain $A$ has a largest element.

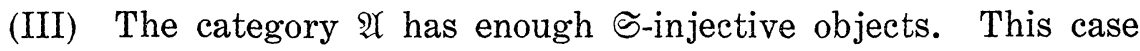
has been dealt with in [3].

1) After submission of this paper to the editor a paper of F. Ulmer Satelliten und derivierte Funktoren, Math. Z. 91 (1966), appeared. Ulmer also uses the method of D. Buchsbaum [1] , and obtains, for the absolute case, results similar to ours. 
In the first paragraph it is shown that the assumptions of (II) resp. (III) are often satisfied. A detailed discussion of the assumption (III) is contained in [3].

The ultimate goal, however not yet attained, of these considerations is to discard the assumption that $\mathfrak{X}$ is additive, and thus to treat functors, e.g. on the category of topological spaces or the category of c.s.s. complexes. There are many examples of nonadditive categories $\mathfrak{A}$ with classes $\mathfrak{S}$ which satisfy the assumptions of (II). We remark that classes $\mathfrak{S}$ with the dual of the above indicated properties are considered in algebraic geometry [6, Ch, 1, p. 139]. This paper only treats right satellites resp. derived functors of covariant functors in one variable, and leaves the obvious generalizations and dualizations to the reader. It should be noted in particular that the special cases treated in $\S 6$ at once dualize to left satellites resp. derived functors including the exactness of the long sequences.

Notational conventions. If $\mathfrak{A}$ is a category and $A, A^{\prime}$ are objects of $\mathfrak{U}$ then $\mathfrak{U}\left(A, A^{\prime}\right)$ is the set of all morphisms from $A$ to $A^{\prime}$. The domain resp. the codomain of a morphism a of $\mathfrak{A}$ are denoted by $Q a$ resp. $Z a$. The class of all morphisms with domain $A$ is denoted by $\mathfrak{A}(A)$. It is preordered in an obvious fashion. If $\subseteq$ is a class of morphisms of $\mathfrak{A}$ we set $\mathfrak{S}(A):=\mathfrak{S} \cap \mathfrak{A}(A)$. The full subcategory of the category of all morphisms of $\mathfrak{U}$ having the elements of $\mathfrak{S}$ as objects is also denoted by $\widetilde{S}$. If $a$ is a morphism with cokernel this cokernel is denoted by $\operatorname{Cok} \alpha$, and the canonical morphism $Z a \longrightarrow \operatorname{Cok} a$ by $\operatorname{cok} a$. Injections of subobjects into objects are often indicated by "inj", canonical projections of direct sums as "proj".

1. The classes $\mathfrak{S}$. 1.1. Definition. Let $\mathfrak{A}$ be a category and $\mathfrak{S}$ a class of morphisms of $\mathfrak{A}$. Denote by $(\mathrm{CO})-(\mathrm{C} 4)$ the following properties of the paị $\mathfrak{A}, \subseteq$ :

(CO) $\mathfrak{A}$ is pointed, and every morphism $0 \stackrel{\mathrm{O}}{\longrightarrow} A$ is contained in $\widetilde{S}$.

(C1) $\mathfrak{S}$ is closed under multiplication, i.e. $a \in \mathfrak{S}, b \in \mathfrak{S}$, and $Q b=Z a$ imply $b a \in \mathfrak{S}$

(C2) $\subseteq$ is closed under base coextension, i.e. if the diagram

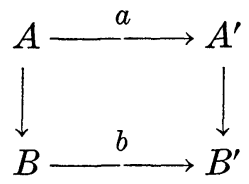

is cocartesian, that is a push-out, and if $a \in \mathfrak{S}$ then $b \in \mathfrak{S}$.

(C3) $\mathfrak{A}$ is pointed, and every element of $\mathfrak{\subseteq}$ has a cokernel. 
(C4) If a product $a b$ is in $\mathfrak{S}$ then $b \in \mathfrak{S}$.

We first discuss some examples of such classes $\mathfrak{S}$, in particular classes which satisfy the properties (II) or (III) of the introduction. We also investigate the question how one can obtain new classes from given ones.

ExAmple [1] 1.2. Let $\mathfrak{A}$ be an Abelian category and $\mathfrak{S}$ a "h.f."class of $\mathfrak{Y}$ in the sense of [1], i.e. let $\subseteq$ be a class of monomorphisms of $\mathfrak{U}$ satisfying

(a) (i) $\subseteq$ is closed under isomorphism.

(ii) All retractions lie in $\subseteq$.

(iii) $\subseteq$ is closed under multiplication.

(iv) If $t$ is a monomorphism and $t s \in \mathfrak{S}$ then $s \in \mathfrak{S}$.

$\left(a^{*}\right)$ The class $\mathfrak{P}(\mathfrak{S})$ of all possible cokernels of elements of $\mathfrak{S}$ satisfies the dual conditions of (i)-(iv).

Then $\mathfrak{S}$ satisfies the properties (CO)-(C3).

If $F: \mathfrak{A} \longrightarrow \mathbb{C}$ is a functor and $\mathfrak{I}$ a class of morphism of $\mathfrak{C}$ then denote by $F^{-1} \mathfrak{I}$ the class of all morphisms of $\mathfrak{U}$ whose image under $F$ lies in $\mathfrak{T}$.

Proposition 1.3. Let $F$ : $\mathfrak{A} \longrightarrow \mathbb{C}$ be a functor which preserves push-outs, and let $\mathfrak{I}$ be a class of morphisms of $\mathfrak{C}$.

(i) If $\mathfrak{I}$ satisfies either one of the conditions (C1), (C2), or (C4) then so does $F^{-1} \mathfrak{T}$.

(ii) If $\mathfrak{A}$, $\mathfrak{C}$, and $F$ are pointed and if $\mathfrak{I}$. satisfies $(\mathrm{CO})$ then so does $F^{-1} \mathfrak{T}$

The proof is obvious.

We remark that $F$, in particular, preserves push-outs if it has a right adjoint.

Proposition 1.4. Let $F: \mathfrak{A} \rightarrow \mathfrak{C}$ be a functor with right adjoint $G: \mathbb{E} \rightarrow \mathfrak{A}$, and let $\mathfrak{I}$ be a class of morphisms of $\mathbb{E}$ satisfying (C4) such that for every object $C$ of $\mathbb{C}$ the class $\mathfrak{I}(C)$ has a largest element. Then, with $\mathfrak{S}:=F^{-1 \mathfrak{T}}$, for every object $A$ of $\mathfrak{A}$ the class $\mathfrak{S}(A)$ has a largest element.

Proof. Let $A$ be any object of $\mathfrak{X}$ and let $t_{0} \in \mathfrak{I}(F A)$ be a largest in $\mathfrak{I}(F A)$. Assume that $s_{0}: A \rightarrow G Z t_{0}$ is the unique morphism such that the diagram

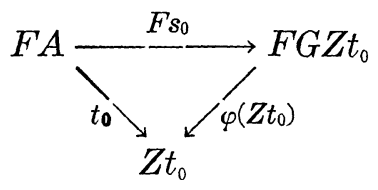


is commutative. In this diagram $\varphi: F G \rightarrow i d_{\Subset}$ is the canonical morphism. Since $\mathfrak{T}$ satisfies $(\mathrm{C} 4)$ and since $t_{0}=\varphi\left(Z t_{0}\right)\left(F s_{0}\right)$ we obtain $F s_{0} \in \mathfrak{T}$ and hence $s_{0} \in \mathfrak{S}$.

We finally show that $s_{0}$ is a largest element of $\mathfrak{S}(A)$. Given $s \in \mathfrak{S}(A)$ there exists $b: F Z s \rightarrow Z t_{0}$ with $b(F s)=t_{0}$. Denoting by

$$
a: Z s \longrightarrow G Z t_{0}
$$

the unique morphism such that

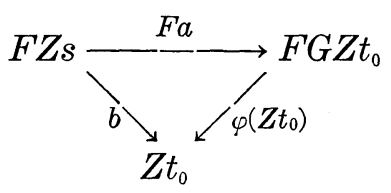

commutes we conclude $t_{0}=\varphi\left(Z t_{0}\right)(F(a s))$ and hence as $=s_{0}$. But this means $s \leqq s_{0}$ in $\subseteq(A)$, and thus $s_{0}$ is a largest element of $\mathfrak{S}(A)$.

The preceding proposition shows that the inverse image under a functor $F$ with right adjoint of a class $\mathfrak{I}$ satisfying (C4) and (II) again satisfies the same conditions. The next propositions state a similar result for the property (III).

DeFinition 1.5. Let $\mathfrak{A}$ be a category, $\subseteq$ a class of morphisms of $\mathfrak{A}$, and $A$ an object of $\mathfrak{A}$. Then $A$ is called S-injective if for all $s \in \mathfrak{S} \mathfrak{\mathfrak { U }}(s, A)$ is surjective.

This notion is due to J. Maranda [7], and has been reintroduced by S. Eilenberg and J. C. Moore in [3] in a slightly different form.

Proposition 1.6 Let $\mathfrak{A}$ be a category, $\mathfrak{M}$ a class of objects of $\mathfrak{A}$, and $\mathfrak{S}(\mathfrak{M})$ the largest class of morphisms of $\mathfrak{A}$ such that all $A \in \mathfrak{M}$ are $\mathfrak{S}$-injective. Then $\mathfrak{S}(\mathfrak{M})$ satisfies (C1), (C2) and (C4). If, in addition, $\widetilde{S}$ is pointed then $(\mathrm{CO})$ is also satisfied.

The proof is obvious.

The preceding proposition implies in particular that the class of morphisms of an injective structure on $\mathfrak{A}$ (in the sense of [7]) satisfies (C1), (C2), (C4). If $\mathfrak{U}$ has enough $\subseteq$-injectives, i.e. if for every

$$
A \in O b \mathfrak{U}
$$

there is an $s \in \mathfrak{S}$ with $\subseteq$-injective $Z s$, then the closure $\bar{\subseteq}$ of $\subseteq$ in the sense of [7] is the class of morphisms of a unique injective structure. Under these hypotheses $\bar{\subseteq}$ coincides with $\subseteq$ if $\subseteq$ satisfies (C4).

The next proposition has first been shown in [3] in the pointed case, and expresses the fact that right adjoints of exact functors preserve injectives. 
Proposition 1.7. Let $F: \mathfrak{A} \rightarrow \mathbb{C}$ be a functor with a right adjoint $G: \mathfrak{C} \rightarrow \mathfrak{A}$, and let $\mathfrak{T}$ be a class of morphisms of $\mathbb{E}$ satisfying (C4) such that $\mathfrak{C}$ has enough $\mathfrak{T}$-injectives. Then with $\mathfrak{S}:=F^{-1} \mathfrak{I}, \mathfrak{A}$ has enough S-injectives.

Proof. Since $F$ and $G$ are adjoint to each other we have

$$
\mathfrak{A}(s, G C) \cong \mathfrak{c}(F s, C)
$$

for every object $C$ in $\mathbb{C}$ and every $s \in \mathfrak{S}$ where the isomorphism is an isomorphism in the category of morphisms of the category of sets. Especially if $C$ is $\mathfrak{I}$-injective then $\mathfrak{S}(F s, C)$ and hence $\mathfrak{A}(s, G C)$ are surjective for every $s \in \mathfrak{S}=F^{-1} \mathfrak{T}$, and $G C$ is $\mathfrak{S}$-injective. Thus $G$ maps $\mathfrak{I}$-injectives to $\mathfrak{S}$-injectives. Finally, if $A$ is an object in $\mathfrak{A}$ and $t \in \mathfrak{I}(F A)$ with $\mathfrak{T}$-injective $Z t$ then the unique morphism

$$
s: A \longrightarrow G Z t
$$

such that the diagram

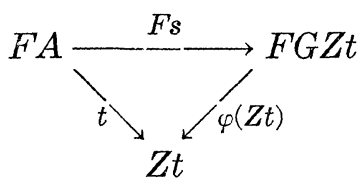

is commutative lies in $\mathfrak{S}$, and $G Z t$ is $\mathfrak{S}$-injective. Hence $\mathfrak{A}$ has enough S-injectives.

Under the assumptions of the preceding proposition both $\mathfrak{S}$ and $\mathfrak{I}$ are the classes of morphisms of injective structures.

At last one can form new classes $\mathfrak{S}$ by means of intersections. In detail we obtain the

Proposition 1.8. Let $\mathfrak{A}$ be a category and $\mathfrak{S}_{\lambda}, \lambda \in A$, a family of classes $\mathfrak{S}_{\lambda}$ of morphisms of $\mathfrak{A}$. Let $\mathfrak{S}_{:}=\bigcap_{\lambda} \mathfrak{S}_{\lambda}$.

(i) If all $\mathfrak{S}_{\lambda}$ satisfy either one of the properties (CO)-(C4) then so does $\mathfrak{S}$.

(ii) If all $\mathfrak{S}_{\lambda}$ satisfy (C4) and (II) resp. (III) and if $\mathfrak{U}$ has direct products then $\mathfrak{S}$ also satisfies (II) resp. (III).

Proof. (i) obvious.

(ii) (a) Assume that all $\mathfrak{S}_{\lambda}$ satisfy (C4) and (II). Let $A \in O b \mathfrak{A}$ and assume that for every $\lambda \in \Lambda s_{\lambda} \in \mathfrak{S}_{\lambda}(A)$ is a largest element. Let $s: A \rightarrow \prod_{\lambda \in \Delta} Z s_{\lambda}$ be the unique morphism with components $s_{\lambda}$. Since all

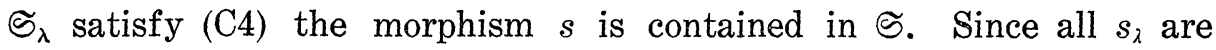
largest elements in $\mathfrak{S}_{\lambda}(A)$ the morphism $s$ is a largest element in $\mathfrak{S}_{(A)}$. Hence $\mathfrak{A}$ satisfies (II). 
(b) Analogously, one shows that (C4) and (III) for the $\mathfrak{\Im}_{2}$ imply (C4) and (III) for $\mathfrak{S .}$

CoROLlary 1.9. Let $\mathfrak{A}$ be a category with direct products,

$$
F_{\lambda}: \mathfrak{A} \longrightarrow \mathfrak{C}_{\lambda}, \lambda \in \Lambda,
$$

a family of functors and for every $\lambda \in \Lambda \mathfrak{I}_{\lambda}$ a class of morphisms of $\mathfrak{F}_{\lambda .}$. Assume that for every $\lambda \in \Lambda$ the hypotheses of 1.4 resp. of 1.7 are satisfied. Then $\mathfrak{S}:=\bigcap_{2} F_{\lambda}^{-1}\left(\mathfrak{I}_{\lambda}\right)$ satisfies (II) resp. (III).

\section{S-exactness.}

DEFINITION 2.1. [8]. Let $\mathfrak{A}$ be a pointed category and $\mathfrak{S}$ a class of morphisms af $\mathfrak{U}$. A sequence $A^{\prime} \stackrel{a}{\longrightarrow} A \stackrel{a^{\prime}}{\longrightarrow} A^{\prime \prime}$ is called S-exact if $\alpha^{\prime} a=0$, if Cok $a$ exists, and if the unique morphism $s: \operatorname{Cok} \alpha \rightarrow A^{\prime \prime}$ with $s(\operatorname{cok} a)=a^{\prime}$ is an element of $\mathfrak{S}$.

If, in particular, $\subseteq$ is of the form $\subseteq(\mathfrak{M})$ where $\mathfrak{M}$ is a class of objects of $\mathfrak{S}$ (see 1.6) and if $A^{\prime} \stackrel{a}{\longrightarrow} A \rightarrow A^{\prime \prime}$ is a complex such that Cok $a$ exists then this sequence is $\subseteq$-exact if and only if for all $M \in \mathfrak{M}$ the sequence

$$
\mathfrak{U}\left(A^{\prime \prime}, M\right) \longrightarrow \mathfrak{U}(A, M) \stackrel{\mathfrak{Y}(a, M)}{\longrightarrow} \mathfrak{X}\left(A^{\prime}, M\right)
$$

is exact in the category of pointed sets. This gives the connection with the exactness definition of [3].

Definition 2.2. Let $\mathfrak{A}$ be a pointed category with a class $\mathfrak{S}$ of morphisms of $\mathfrak{A}$ satisfying $(\mathrm{C} 3)$, and $T: \mathfrak{A} \rightarrow \mathfrak{B}$ a functor into an Abelian category $\mathfrak{B}$. Then $T$ is called $\mathfrak{S}$-half resp. $\subseteq$-left exact if for every $s \in \subseteq$ the sequences

$$
T Q s \stackrel{T s}{\longrightarrow} T Z s \stackrel{\text { Teoks }}{\longrightarrow} T \text { Coks }
$$

resp.

$$
0 \longrightarrow T Q s \stackrel{T s}{\longrightarrow} T Z s \stackrel{T \text { coks }}{\longrightarrow} T \text { Coks }
$$

are exact.

If $T$ is $\mathfrak{S}$-left exact then $T$ transforms every $\mathfrak{S}$-exact sequence

$$
0 \longrightarrow A^{\prime} \longrightarrow A \longrightarrow A^{\prime \prime}
$$

into an exact sequence

$$
0 \longrightarrow T A^{\prime} \longrightarrow T A \longrightarrow T A^{\prime \prime}
$$


If $T$ transform $\mathfrak{S}$ into Mon $\mathfrak{B}$ (the class of all monomorphisms of $\mathfrak{B}$ ) then $T A=0$ for every $A$ in $\mathfrak{U}$ such that $0: A \rightarrow 0$ is in $\subseteq$.

For the remainder of this paragraph we assume that $\mathfrak{A}$ is an additive category ${ }^{2)}$ and $\subseteq$ a class of morphisms of $\mathfrak{A}$ satisfying (CO)(C3).

We list some properties of $\subseteq$ and $\mathfrak{\subseteq}$-exactness which will be used later on.

2.3. If $a$ is any morphism of $\mathfrak{A}$ then its graph

$$
\Gamma_{a}:=(i d, a): Q a \longrightarrow Q a \oplus Z a
$$

is an element of $\mathfrak{\subseteq}$. This follows from the fact that the diagram

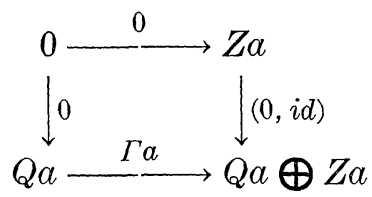

is cocartesian. Especially for every $A$ and $A^{\prime}$ in $O b \Re$ the canonical injection (id, 0$): A \rightarrow A \oplus A^{\prime}$ is in $\widetilde{\subseteq}$. Also all isomorphisms lie in $\subseteq$.

2.4. If $s$ and $t$ are in $\subseteq$ then so is $s \oplus t$. Since

$$
(s \oplus t)=(s \oplus i d)(i d \oplus t)
$$

and by (C1) we need only show $s \oplus i d \in \mathfrak{S}$. But for every $A \in O b \mathfrak{A}$ the diagram

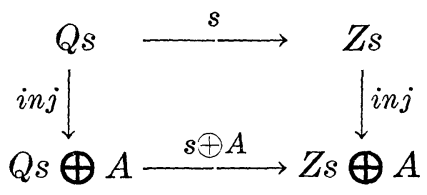

is cocartesian

First IsOMORPhism Theorem 2.5. Consider a commutative

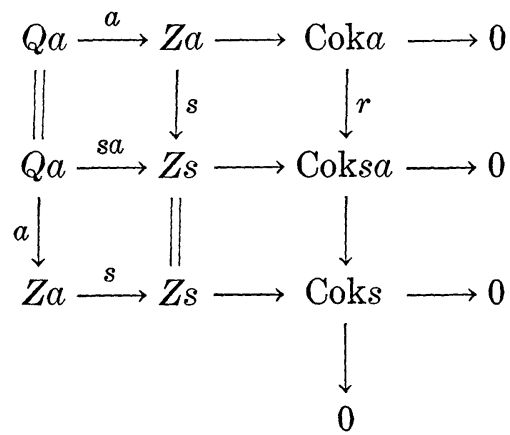

2) As in [5] we assume that there are finite direct sums in an additive category. 
with exact rous and $s \in \mathfrak{S}$. Then $r \in \mathfrak{S}$ and the last column is exact. The exactness of the last column is standard. The upper right square is cocartesian, and hence $r \in \mathfrak{S}$ since $s \in \mathfrak{S}$.

2.6. Let $A \in O b \mathfrak{H}, a \in \mathfrak{U}(A)$, and $s \in \mathfrak{S}(A)$. Consider the exact sequence

$$
A \stackrel{(a, s)}{\longrightarrow} Z a \bigoplus Z s \stackrel{\left(\mu_{1}, \mu_{2}\right)}{\longrightarrow} \operatorname{Cok}(a, s) \longrightarrow 0 .
$$

(The existence of $\operatorname{Cok}(a, s)$ follows from (C3) since $(a, s) \in \mathbb{S}$.)

Then $\mu_{1} \in \mathfrak{S}$.

This follows from the cocartesian diagram

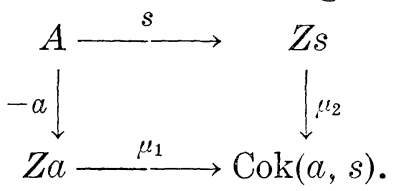

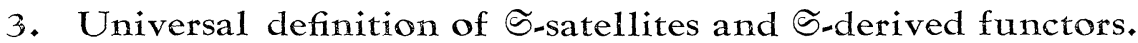
Let $\mathfrak{i}$ be a pointed category and $\subseteq$ a class of morphisms of $\mathfrak{A}$ satisfying (C3) with $i d_{A} \in \mathfrak{S}$ for every $A \in O b 2$. We denote by $Q$ resp. $Z$ resp. Cok the functors from the category $\mathfrak{S}$ into $\mathfrak{U}$ mapping an $s \in \mathfrak{S}$ to its domain $Q s$ resp. its codomain $Z s$ resp. its cokernel Coks. Let $\mathfrak{B}$ be an Abelian category.

An $\subseteq$ - $\delta$-functor (see [5], Ch. 2) is a tripel $\hat{T}=\left(T^{0}, T^{1}, \delta_{\hat{r}}\right)^{3)}$ of pointed functors $T^{0}, T^{1}: \mathfrak{U} \rightarrow \mathfrak{B}$ and a functorial morphism

$$
\delta_{\hat{T}}: T^{0} \mathrm{Cok} \rightarrow T^{1} Q
$$

such that the sequence

$$
T^{0} Z \longrightarrow T^{0} \mathrm{Cok} \stackrel{\delta_{\hat{T}}}{\longrightarrow} T^{1} Q \longrightarrow T^{1} Z
$$

is a complex. The $\subseteq-\delta$-functors form a category in an obvious manner. An $\mathfrak{S}$ - $\delta$-functors $\hat{T}=\left(T^{0}, T^{1}, \delta_{\hat{T}}\right)$ is called universal if for every $\mathfrak{S}-\delta$-functor $\hat{U}=\left(U^{0}, U^{1}, \delta_{\hat{U}}\right)$ and every morphism $b^{0}: T^{0} \rightarrow U^{0}$ there is a unique morphism $\hat{b}: \hat{T} \rightarrow \hat{U}$ extending $b^{0}$. If $\hat{T}=\left(T^{0}, T^{1}, \delta_{\hat{T}}\right)$ is universal then $\hat{T}$ is determined by $T^{0}$ and by its universal property up to isomorphism. For every pointed functor $T: \mathfrak{U} \rightarrow \mathfrak{B}$ for which there exists a universal $\mathfrak{S}-\delta$-functor with first component $T$ we choose a particular one, $\left(T, S^{1} T, \delta_{T^{\prime}}\right)$, and call $S^{1} T$ the first satellite of $T$. The maps

3) Here and at other places of this paragraph and of the whole paper one encounters the usual set-theoretical difficulties which can be avoided by the customary methods. 


$$
T m\left(T, S^{1} T, \delta_{T}\right) \text { and } T m S^{1} T
$$

are functors ${ }^{3)}$ defined on a suitable full subcategory of the category of all pointed functors from $\mathfrak{A}$ to $\mathfrak{B}$. Inductively or by a corresponding universal property we define the higher satellites $S^{n} T$ of $T$. We call an $\mathfrak{S}-\delta$-functor $\left(T^{0}, T^{1}, \delta\right) \mathfrak{S}$-exact if for every $s \in \mathfrak{S}$ the sequence

$$
T^{0} Q s \longrightarrow T^{0} Z s \longrightarrow T^{0} \mathrm{Coks} \stackrel{\delta(s)}{\longrightarrow} T^{1} Q s \longrightarrow T^{1} Z s \longrightarrow T^{1} \mathrm{Coks}
$$

is exact in $\mathfrak{B}$.

A triple $\widehat{T}=\left({ }_{0} T,{ }_{1} T, \rho_{\hat{T}}\right)$ of pointed functors ${ }_{0} T,{ }_{1} T: \mathfrak{A} \rightarrow \mathfrak{B}$ and a morphism $\rho_{\hat{T}}:{ }_{0} T \rightarrow{ }_{1} T$ is called a $\subseteq-\rho$-functor if for every $s \in \mathfrak{S}$ the sequence

$$
\mathrm{Ke}_{0} T s \longrightarrow{ }_{0} T Q s \stackrel{\rho_{\hat{T}}(s)}{\longrightarrow}{ }_{1} T Q s
$$

is a complex. The $\subseteq-\rho$-functors again form a category with obvious morphisms. An $\subseteq-\rho$-functor $\widehat{T}=\left({ }_{0} T,{ }_{1} T, \rho_{\hat{T}}\right)$ is called universal if for every $\subseteq$ - $\rho$-functor $\hat{U}=\left({ }_{0} U,{ }_{1} U, \rho_{\hat{U}}\right)$ and every morphism

$$
{ }_{0} b:{ }_{0} T \longrightarrow{ }_{0} U
$$

there is a unique extension $\hat{b}: \hat{T} \rightarrow \hat{U}$ of ${ }_{0} b$. If $\hat{T}=\left({ }_{0} T,{ }_{1} T, \rho_{\hat{T}}\right)$ is universal then $\hat{T}$ is determined up to isomorphism by ${ }_{0} T$ and its universal property. For every pointed functor $T: \mathfrak{A} \rightarrow \mathfrak{B}$ for which there is a universal $\subseteq$ - $\rho$-functor with first component $T$ we choose a particular one, $\left(T, M T, \rho_{T}\right)$, and obtain the functor $T \rightarrow\left(T, M T, \rho_{T}\right)$. The pair $\left(M T, \delta_{T^{\prime}}\right)$ has, in particular, the universal property that every morphism $f: T \rightarrow T^{\prime}$ where $T^{\prime}$ transforms $\subseteq$ into Mon $\mathfrak{B}$ factors uniquely through $\rho_{T^{\prime}}$. If $M T$ itself maps $\mathfrak{S}$ into Mon $\mathfrak{B}$ then the pair $\left(M T, \rho_{T}\right)$ is determined, by both $T$ and the latter universal property, up to isomorphism, and we obtain the functor

$$
M^{0}: T \sim\left(M T, \rho_{T}\right)
$$

mapping any $T$ such that $\left(T, M T, \rho_{T}\right)$ exists and such that $M T$ transforms $\mathfrak{S}$ into Mon $\mathfrak{B}$ to $\left(M T, \rho_{T}\right)$. In this case $M T$ is called the universal $\mathfrak{S}$-monofunctor of $T$.

Finally, we call a triple

$$
\hat{T}=\left(T_{0}, T_{1}, \varphi_{\hat{T}}\right)
$$

an $\mathfrak{S}-\varphi$-functor if $T_{0}, T_{1}: \mathfrak{A} \rightarrow \mathfrak{B}$ are pointed functors, if

$$
\varphi_{\hat{T}}: \operatorname{Ke} T_{0} \operatorname{cok} \rightarrow T_{1} Q
$$

is a morphism of functors defined on $\mathfrak{S}$. Since for every morphism $a: A \rightarrow B$ the diagram 


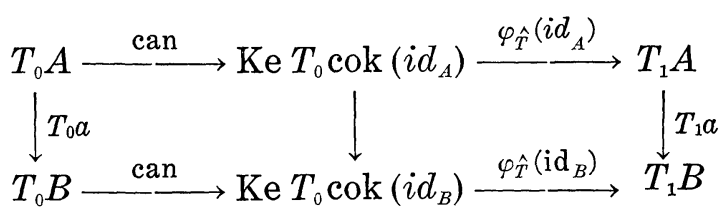

is commutative $\varphi_{\hat{T}}$ gives rise to a morphism $\widetilde{\varphi}_{\hat{T}}: T_{0} \rightarrow T_{1}$. Again, the $\subseteq-\varphi$-functors form a category, and we have the notion of a universal $\mathfrak{S}-\varphi$-functor. For every pointed functor $T: \mathfrak{A} \rightarrow \mathfrak{B}$ for which there is a universal $\subseteq-\varphi$-functor with first component $T$ we choose a particular one, $\left(T, R T, \varphi_{T}\right)$, and obtain the functor $T \leadsto\left(T, R T, \varphi_{T}\right)$. Moreover the pair $\left(R T, \widetilde{\varphi}_{T}\right)$ has the universal property that every morphism $f: T \rightarrow T^{\prime}$ where $T^{\prime}$ is $\widetilde{S}$-left exact factors uniquely through $\varphi_{T}$. If $R T$ itself is $\widetilde{S}_{\text {-left }}$ exact then $\left(R T, \widetilde{\varphi}_{T^{\prime}}\right)$ is determined up to isomorphism by both $T$ and the last universal property. In this case

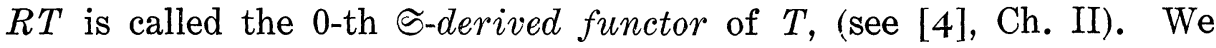
obtain the functor

$$
R^{\circ}: T \leadsto R T
$$

mapping any $T$ such that $\left(T, R T, \varphi_{T^{\prime}}\right)$ exists and that $R T$ is S-left exact to $R T$. The satellite $R^{n} T:=S^{n} R^{\circ} T$ of $R^{\circ} T$ is called the $n$-th derived functor of $T$. If $M^{\circ} T$ and $R^{\circ} M T$ exist then $R^{\circ} M^{\circ} T=R^{\circ} T$. This remark is usefull since we can often show the existence of $M^{\circ} T$ and that of $R^{\circ} T^{\prime}$ provided $T^{\prime}$ transforms $\subseteq$ into Mon 2 .

Proposition 3.1. The functors

$$
T \leadsto\left(T, S^{1} T, \delta_{T}\right) \text { resp. } T \leadsto\left(T, M T, \rho_{T^{\prime}}\right) \text { resp. } T \leadsto\left(T, R T, \varphi_{T^{\prime}}\right)
$$

are left adjoint to the functors which map any $\subseteq-\delta$-resp. $\subseteq-\rho$ resp. $\subseteq-\varphi$-functor into its first component. Hence they commute with direct limits. The same holds true for the functors

$$
M^{\circ}, R^{n}, S^{n}, n \geqq 0 .
$$

All the above introduced functors depend functorially on $\subseteq$. We use an index to distinguish notions with respect to different classes $\mathfrak{S}$.

If $\mathfrak{A}$ is additive we complete the above definitions by requiring that the functors are additive.

\section{Existence theorems.}

REMARK 4.1. Let $\mathfrak{B}$ be an Abelian category and $I$ a preordered class. We do not assume that $I$ is a set. Nevertheless direct systems $\widehat{B}=\left(B_{i}, \beta_{j i}\right)$ over $I$ of objects $B_{i}, i \in I$, in $\mathfrak{B}$ and morphisms 


$$
\beta_{j i}: B_{i} \longrightarrow B_{j}, i \leqq j,
$$

with the usual compatibility properties are well-defined. If an object $B$ together with morphisms $\beta_{i}: B_{i} \rightarrow B$ has the universal property of the direct limit we write

$$
\left(B, \beta_{i}\right)=\underset{I}{\stackrel{\lim }{\longrightarrow}}\left(B_{i}, \beta_{j i}\right) .
$$

The map $\hat{B} \leadsto B$ is again a functor. ${ }^{3)}$

The reason for considering such direct limits over classes is that we do not want to restrict ourselves to small domain categories as is done in [1] since for applications smallness is impractical.

For the remainder of this paper we assume that $\mathfrak{A}$ is an additive category, $\subseteq$ a class of morphisms of $\mathfrak{A}$ satisfying (CO)-(C3), and $\mathfrak{B}$ an Abelian category. All functors are additive functors from $\mathfrak{A}$ to $\mathfrak{B}$.

Let $T: \mathfrak{A} \rightarrow \mathfrak{B}$ be an additive functor. For $s \in \mathfrak{S}$ one has the exact sequence

$$
Q s \stackrel{s}{\longrightarrow} Z s \stackrel{\text { can }}{\longrightarrow} \text { Coks } \longrightarrow 0 \text {. }
$$

This sequence gives rise to a commutative exact diagram

$$
\begin{aligned}
& 0 \rightarrow \mathrm{Ke} T s \stackrel{\text { inj }}{\longrightarrow} T Q s \stackrel{\text { can }}{\longrightarrow} \text { Coim } \mathrm{Ts} \rightarrow 0
\end{aligned}
$$

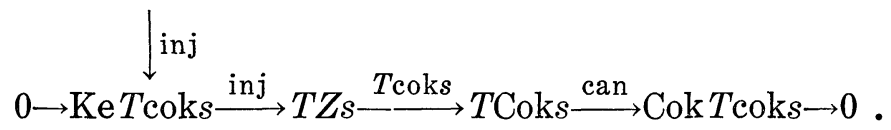

We define

$$
{ }_{s} T:=\operatorname{Coim} T s T_{s}:=\operatorname{Ke} T \operatorname{cok} s, \text { and } T^{s}:=\operatorname{Cok} T \operatorname{cok} s .
$$

LEMMA 4.2. (i) Let $s, t \in \subseteq$ and $a \in \mathfrak{A}(Q s, Q t)$ such that $s \leqq t a$ in $\mathfrak{A}(Q s)$. Then there are unique morphisms

$$
{ }_{t s} T(a):{ }_{s} T \rightarrow{ }_{t} T, T_{t s}(a): T_{s} \rightarrow T_{t} \text {, and } T^{t s}(a): T^{s} \rightarrow T^{t}
$$

such that for all $b$ with $b s=t a$ the diagram

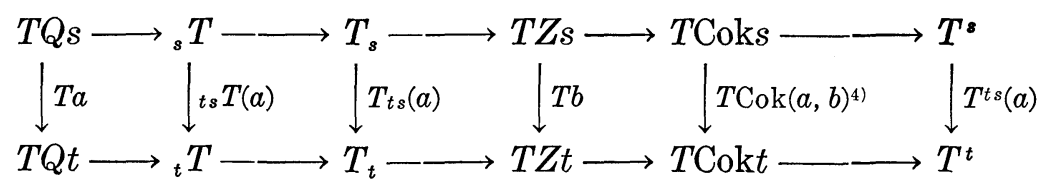

is commutative.

3) Here $(a, b) \in \subseteq(s, t)$ and hence $\operatorname{Cok}(a, b)$ is the unique morphism with $\operatorname{Cok}(a, b)$ $\operatorname{cok} s=(\operatorname{cok} t) b$. 
(ii) Assume in addition $u \in \mathfrak{S}$ and $a^{\prime} \in \mathfrak{A}(Q t, Q u)$ with $t \leqq u a^{\prime}$. Then the compatibility conditions

$$
\begin{aligned}
& { }_{u t} T\left(a^{\prime}\right)_{t s} T(a)={ }_{u s} T\left(a^{\prime} a\right),{ }_{s s} T\left(i d_{Q s}\right)=i d \\
& T_{u t}\left(a^{\prime}\right) T_{t s}(a)=T_{u s}\left(a^{\prime} a\right), T_{s s}\left(i d_{Q s}\right)=i d \\
& T^{u t}\left(a^{\prime}\right) T^{t s}(a)-T^{u s}\left(a^{\prime} a\right), T^{s s}\left(i d_{Q_{s}}\right)=i d
\end{aligned}
$$

hold.

The proof is essentially contained in [2, Ch. 3, pp. 34-35]. In particular, for every $A \in O b \mathfrak{A}$ and $s \leqq t \in \subseteq(A)$ we define

$$
{ }_{t s} T:={ }_{t s} T\left(i d_{A}\right), T_{t s}:=T_{t s}\left(i d_{A}\right), T^{t s}:=T^{t s}\left(i d_{A}\right)
$$

and thus obtain the direct systems

$$
\left({ }_{s} T,{ }_{t s} T, s \in \mathfrak{S}(A)\right),\left(T_{s}, T_{t s}, s \in \mathfrak{S}(A)\right) \text {, and }\left(T^{s}, T^{t s}, s \in \mathfrak{S}(A)\right)
$$

over the preordered class $\mathfrak{S}(A)$.

We first investigate the existence of the first satellite and prove a theorem generalizing and sharpening the existence theorem of [1]. If $\hat{T}=\left(T, T^{1}, \delta\right)$ is an $\subseteq-\delta$-functor denote by $\hat{T}^{s}, s \in \mathfrak{S}$, the unique morphism satisfying $\hat{T}^{s}(\operatorname{cok} T \operatorname{cok} s)=\delta(s)$.

Obviously one has $\hat{T}^{t} T^{t s}=\widehat{T}^{s}$ for $s \leqq t$.

TheOREM 4.3 [8]. (i) Let $\widehat{T}=\left(T, T^{1}, \delta\right)$ be an $\subseteq-\delta$-functor, and assume that for all $A \in \mathrm{Ob \mathfrak {A }}$

$$
\left(T^{1} A, \hat{T}^{s}\right)=\underset{\lim }{\longrightarrow}\left(T^{s}, T^{t s}, s \in \mathfrak{S}(A)\right) .
$$

Then $\hat{T}$ is a universal $\subseteq-\delta$-functor.

(ii) Let $T$ be a functor, and assume that for every $A \in \mathrm{ObN}$ the direct limit of the system $\left(T^{s}, T^{t s}, s \in \mathfrak{S}(A)\right)$ exists. Define

$$
\begin{aligned}
& \left(T^{1} A, \hat{T}^{s}\right):=\underset{\lim }{\longrightarrow}\left(T^{s}, T^{t s}, s \in \mathfrak{S}(A)\right) \\
& \delta(s):=\widehat{T}^{s}(\operatorname{cok} T \operatorname{cok} s) \text { and } \delta:=(\delta(s))_{s \in \subseteq} \text {. }
\end{aligned}
$$

Then $T^{1}$ becomes a functor in a natural way such that $\left(T, T^{1}, \delta\right)$ is a universal $\subseteq-\delta$-functor, i.e. the first satellite $S^{1} T$ of $T$ exists.

Proof. (i) Let $U=\left(U, U^{1}, \delta\right)$ be any $\mathfrak{S}-\delta$-functor, and let $b$ : $T \rightarrow U$ be a morphism. The existence of a unique $b^{1}: T^{1} \rightarrow U^{1}$ such that $\left(b, b^{1}\right): \hat{T} \rightarrow \hat{U}$ is a morphism has to be shown.

For all $A \in \mathrm{ObS}$ and $s \leqq t$ in $\subseteq(A)$ we obtain a commutative diagram 


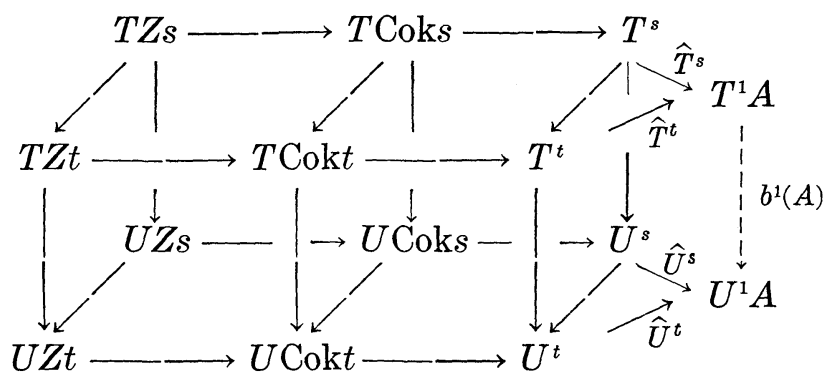

in which the vertical morphisms of the back-resp. front surface are $b(Z s), b(\operatorname{Coks})$ and $\operatorname{Cok}(b(Z s), b(\operatorname{Cok} s))$ resp. $b(Z t), b(\operatorname{Cok} t)$, and $\operatorname{Cok}(b(Z t)$, $b((\operatorname{Cok} t))$ and in which $b^{1}(A)$ is the unique morphism with

$$
b^{1}(A) \widehat{T}^{s}=\hat{U}^{s} \operatorname{Cok}(b(Z s), b(\operatorname{Coks})) \text { for all } s \in \mathfrak{S}(A) .
$$

The unique existence of $b^{1}(A)$ is a consequence of condition 4.4.

But $b^{1}:=\left(b^{1}(A)\right)_{A \in o b \mathfrak{U}}$ is indeed a functorial morphism. For let $a \in \mathfrak{A}(A, B)$. For every $s \in \mathfrak{S}(A)$ we choose a commutative diagram

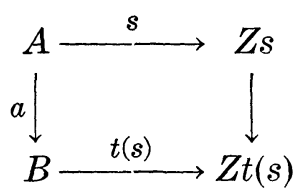

with $t(s) \in \mathfrak{S}(B)$. The existence of such a diagram follows from 2.6. We then obtain diagrams

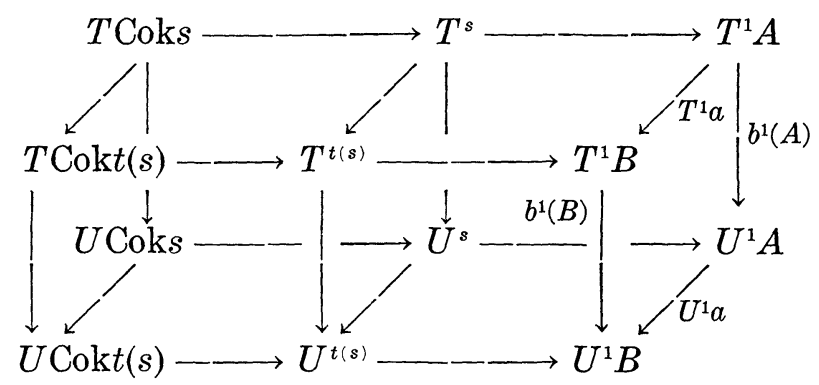

in which all partial squares up to that of the right hand surface are commutative by construction. Especially

$$
b^{1}(B)\left(T^{1} a\right) \widehat{T}^{s}=\left(U^{1} a\right) b^{1}(A) \widehat{T}^{s} \text { for all } s \in \mathfrak{S}(A)
$$

which again implies

$$
\begin{aligned}
& \left.b^{1}(B)\left(T^{1} a\right)=\left(U^{1} a\right) b^{1}(A) \text { by } 4.4\right) \\
& \text { i.e. } b^{1}: T^{1} \longrightarrow U^{1}
\end{aligned}
$$

is a morphism. 
The compatability of $\left(b, b^{1}\right)$ with $\delta$ follows from the construction, the uniqueness of $b^{1}$ is again a consequence of 4.4.

(ii) This part of the theorem is proven with the same method as in (i).

Under stronger assumptions on $\mathfrak{S}$ the preceding result can be sharpened.

Proposition 4.5. [8]. Let $T=\left(T, T^{1}, \delta\right)$ be an exact $\subseteq-\delta$-functor. Assume that $\mathfrak{B}$ has exact filtered direct limits ((AB5) of [5]), and that the classes of subobjects of the objects of $\mathfrak{B}$ are sets. Then $T^{1}$ is universal if and only if for all $A \in \mathrm{Ob \Re}$ the relation

$$
T^{1} A=\operatorname{Sup}_{s \in \mathbb{S}(A)} \operatorname{Im} \delta(s)
$$

holds.

Proof. We remark first that under the assumptions of the proposition the supremum of a family of subobjects $B_{\lambda}$ of a given object $B$ of $\mathfrak{B}$, indexed by any class $A$, exists. Define namely $B_{\Delta^{\prime}}=\operatorname{Sup}_{\lambda \in \Delta^{\prime}} B_{\lambda}$ for every subset $\Lambda^{\prime} \subset \Lambda$. Then from obvious set theoretical reasons, these $B_{\Delta}$, become stationary, i.e, there is a subset $\Lambda_{0}^{\prime}$ of $\Lambda$ such that $B_{\Delta^{\prime}}=B_{\Delta_{0}^{\prime}}$ for every $\Lambda^{\prime} \geqq \Lambda_{0}^{\prime}$. This $B_{\Delta_{0}^{\prime}}$ is the supremum of the $B_{\lambda}$. Especially, $\operatorname{Sup}_{s \in \mathbb{S}(A)} \operatorname{Im} \delta(s)$ exists in $T^{1} A$.

Using this remark and that for every $A \in \mathrm{ObO}$ there is a filtered subset $\mathfrak{S}^{\prime}(A)$ of $\mathfrak{S}(A)$ such that

$$
\begin{aligned}
& \operatorname{Sup}_{s \in \mathbb{S}^{(A)}} \operatorname{Im} \delta(s)=\operatorname{Sup}_{s \in \mathbb{S}^{\prime}(A)} \operatorname{Im} \delta(s) \\
= & \lim _{\mathrm{\longrightarrow}}\left(\operatorname{Im} \delta(s), \operatorname{inj}, s \in \mathfrak{S}^{\prime}(A)\right) \\
= & \underset{\mathrm{lim}}{\longrightarrow}\left(T^{t}, T^{t s}, s \in \mathfrak{S}^{\prime}(A)\right) \\
= & \stackrel{\lim }{\longrightarrow}\left(T^{t}, T^{t s}, s \in \mathfrak{S}(A)\right)
\end{aligned}
$$

the asserted result at once follows from the preceding proposition.

In a similar fashion we obtain existence theorems for $\subseteq-\rho$-functors. If $\hat{T}=\left(T,{ }_{1} T, \rho\right)$ is an $\mathscr{S}-\rho$-functor and $s \in \mathfrak{S}$ denote by ${ }_{8} \hat{T}$ the unique morphism rendering

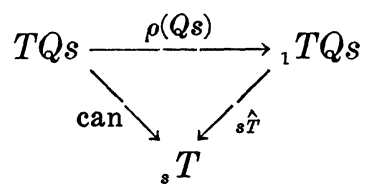

commutative. For $s \leqq t$ in $\subseteq(A)$ we obviously have ${ }_{t} \widehat{T}_{t s} T={ }_{s} T$. 
THEOREM 4.6. (i) Let $\hat{T}=\left(T,{ }_{1} T, \rho\right)$ be an $\subseteq-\rho$-functor. Then $T$ is universal if for all $A \in \mathrm{Ob}$

$$
\left.{ }_{1} T A,{ }_{s} \hat{T}, s \in \mathfrak{S}(A)\right)=\underline{\lim }\left({ }_{s} T,{ }_{t s} T, s \in \mathfrak{S}(A)\right) .
$$

(ii) Let $T$ be a functor. Assume that for every $A \in \mathrm{ObN}$ the direct limit of $\left({ }_{s} T,{ }_{t s} T, s \in \mathfrak{S}(A)\right)$ exists. Define

$$
\left(M T A,{ }_{s} \hat{T}, s \in \mathfrak{S}(A)\right)=\underline{\lim }\left({ }_{s} T,{ }_{t s} T, s \in \mathfrak{S}(A)\right)
$$

$\rho(A):={ }_{s} \hat{T} \operatorname{can}_{s}$ where $s$ is any element of $\mathfrak{S}(A)$ and $\operatorname{can}_{s}: T A \rightarrow{ }_{s} T$, and $\rho:=(\rho(A))_{A \in \text { obgr. }}$ Then $M T$ is a functor in a natural way such that $(T, M T, \rho)$ is a universal $\subseteq$ - $\rho$-functor.

The proof is analogous to that of Theorem 4.3 and left to the reader. The assumptions of Theorem 4.6 can be verified under additional assumptions on $\mathfrak{B}$.

Proposition 4.7. Let $T$ be a functor.

(i) Assume that for every $A \in$ ObभI the supremum of the subojects $\mathrm{Ke} T s, s \in \mathfrak{S}(A)$, of $T A$ exists, and that for every morphism $b: T A \rightarrow B$

$$
b\left(\operatorname{Sup}_{s} \operatorname{Ke} T s\right)=\operatorname{Sup}_{s} b(\operatorname{Ke} T s)
$$

holds. Then the direct limit $M T A$ of $\left({ }_{s} T,{ }_{t s} T, s \in \mathcal{S}(A)\right)$ exists, and is equal to $T A / \mathrm{Sup}_{s} \mathrm{Ke} T s$.

(ii) Assume in addition to the hypotheses of (i) that for every $a \in \mathfrak{S}$ we have

$$
\left.(T a)^{-1} \operatorname{(Sup}_{s \in \mathbb{E}(Z a)} \operatorname{Ke} T s\right)=\operatorname{Sup}_{s \in \mathbb{S}(Z a)}(T a)^{-1}(\operatorname{Ke} T s) .
$$

Then the functor $M T$ transforms $\subseteq$ into Mon $\mathfrak{B}$.

REMARK 4.8. The assumptions of (i) always hold if $\mathfrak{B}$ has arbitrary direct sums, and the classes of subobjects of objects of $\mathfrak{B}$ are sets. If in addition $\mathfrak{B}$ has exact filtered direct limits then also (ii) holds.

Proof of Prop. 4.7. (i) For $A \in \mathrm{ObO}$ and $t \in \mathfrak{A}(A)$ consider the commutative exact diagram

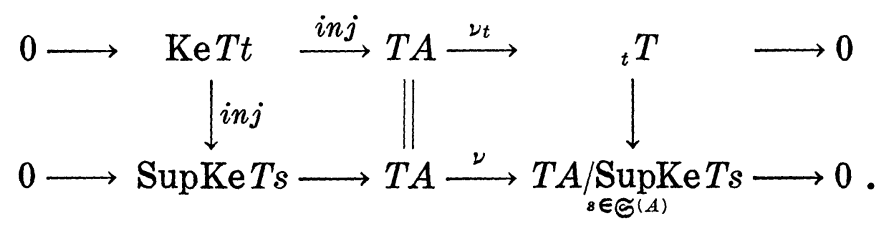

Let then $b_{s}:{ }_{s} T \rightarrow B$ be a family of morphisms compatible with the ${ }_{t s} T$. These $b_{s}$ at once determine a $b^{\prime}: T A \longrightarrow B$ such that $b^{\prime}=b_{s} \nu_{s}$, 
and hence $b^{\prime}(\operatorname{Ke} T s)=0$ for every $\mathrm{s} \in \subseteq(A)$. This implies

$$
b^{\prime}\left(\operatorname{SupKe}_{s} T s\right)=\operatorname{Sup}_{s} b^{\prime}(\operatorname{Ke} T s)=0 .
$$

Therefore there is a unique morphism $b$ with $b^{\prime}=b \nu$. Hence

$$
T A / \operatorname{SupKe}_{s} T s=\stackrel{\lim }{\longrightarrow}\left({ }_{s} T,{ }_{t s} T, s \in \subseteq(A)\right) .
$$

(ii) We remark first that

$$
(T a)^{-1}(\operatorname{Ke} T s)=\operatorname{Ke} T s a \text { for } s \in \mathfrak{S}(Z a),
$$

and that the class $\{s a \mid s \in \mathfrak{S}(Z a)\}$ is cofinal in $\mathfrak{S}(Q a)$, so

$$
\operatorname{Sup}_{s \in \mathbb{S}(Z a)}(T a)^{-1}(\operatorname{Ke} T s)=\operatorname{Sup}_{t \in \mathbb{S}(Q a)} \operatorname{Ke} T t .
$$

But the condition

$$
(T a)^{-1}\left(\operatorname{Sup}_{s} \operatorname{Ke} T s\right)=\operatorname{Sup}_{s}(T a)^{-1}(\operatorname{Ke} T s)
$$

obviously implies that

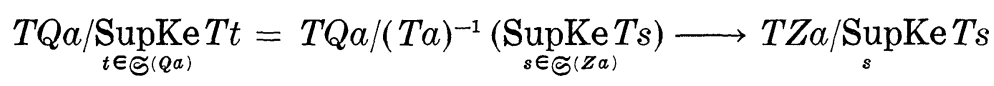

is a monomorphism, hence the assertion.

The important feature of the preceding theorem is that the existence of $M T$ can always be shown if only $\mathfrak{B}$ has good enough properties which is mostly the case in applications.

Finally we obtain the existence theorem for $\mathfrak{S}-\phi$-functors.

THEOREM 4.9 (i) An $\subseteq-\varphi$-functor $\left(T, T_{1}, \varphi\right)$ is universal if for all $A \in \mathrm{ObN}$,

$$
\left(T_{1} A, \varphi(s), s \in \mathfrak{S}(A)\right)=\underline{\lim }\left(T_{s}, T_{t s}, s \in \mathfrak{S}(A)\right) .
$$

(ii) Let $T$ be a functor. Assume that for every $A \in \mathrm{ObN}$ the direct limit of $\left(T_{s}, T_{t s}, s \in \mathfrak{A}(A)\right)$ exists, and denote it by $(R T A, \varphi(s)$, $s \in \mathfrak{X}(A))$, and $\varphi:=(\varphi(s))_{s \in \mathbb{S}}$.

Then $R T$ becomes a functor in a natural way such that $(T, R T, \varphi)$ is a universal $\subseteq-\varphi$-functor.

Again the proof is analogous to that of Theorem 4.3.

5. Exactness. Again we are given $\mathfrak{A}$, $\subseteq$ and $\mathfrak{B}$ as in $\S 4$. Assume that in addition to the class $\subseteq$ we are given a subclass $\mathfrak{S}^{\prime}$ of $\subseteq$ with the following two properties:

$\left(\mathrm{C}^{\prime} 1\right)$ For every $A \in \mathrm{Ob} \mathfrak{A}$ the class $\mathfrak{S}^{\prime}(A)$ is cofinal in $\mathfrak{S}(A)$.

(C'2) If $s \in \mathfrak{S}^{\prime}(A), t \in \mathfrak{S}(A)$, and $s \leqq t$, then $t \in \mathfrak{S}^{\prime}(A)$. Assume now that $T: \mathfrak{A} \rightarrow \mathfrak{B}$ is an additive functor such that for every $A \in \mathrm{ObO}$ the direct limit of $\left(T^{s}, T^{t s}, s \in \mathfrak{S}(A)\right)$ exists. 
Definition 5.1. The functor $\lim$ is called $\left(T, \mathfrak{S}^{\prime}, \delta\right)$-exact ${ }^{5)}$ if the following condition holds: Given the following data

(a) a complex $A_{0} \stackrel{a_{1}}{\longrightarrow} A_{1} \stackrel{a_{2}}{\longrightarrow} A_{2}$

(b) a filtered class $I$

(c) order preserving maps

$$
\alpha_{\lambda}: I \longrightarrow \mathfrak{S}\left(A_{\lambda}\right), \lambda=0,1,2
$$

satisfying

(i) $\quad \alpha_{\lambda-1}(i) \leqq \alpha_{\lambda}(i) a_{\lambda}$ for $\lambda=1,2$ and $i \in I$

(ii) $T^{\alpha_{0}(i)} \stackrel{T^{\alpha_{1}(1) \alpha_{0}(i)}\left(a_{1}\right)}{\longrightarrow} T^{\alpha_{1}(i)} \stackrel{T^{\alpha_{2}(i) \alpha_{1}(i)}\left(a_{2}\right)}{\longrightarrow} T^{\alpha_{2}(i)}$ exact for $i \in I$

(iii) the images $\operatorname{Im} \alpha_{\lambda}$ of all three $\alpha_{\lambda}$ are cofinal and contained in $\mathfrak{S}^{\prime}$, or $\operatorname{Im} \alpha_{1}$ and $\operatorname{Im} \alpha_{2}$ are cofinal and contained in $\mathfrak{S}^{\prime}$ and $\left|\operatorname{Im} \alpha_{0}\right|=1$, or $\operatorname{Im} \alpha_{2}$ is cofinal and contained in $\mathfrak{S}^{\prime},\left|\operatorname{Im} \alpha_{1}\right|=1$, and $A_{0}=0$ then also the sequence $\lim _{\rightarrow} T^{\alpha_{0}(i)} \rightarrow \underset{\rightarrow}{\lim } T^{\alpha_{1}(i)} \rightarrow \underset{\rightarrow}{\lim } T^{\alpha_{0}(i)}$ is exact.

THEOREM 5.2. Let $T: \mathfrak{A} \rightarrow \mathfrak{B}$ be an additive functor and $\mathfrak{S}^{\prime} a$ subclass of satisfying $\left(\mathrm{C}^{\prime} 1\right)$ and $\left(\mathrm{C}^{\prime} 2\right)$. Assume that

(i) for every $A \in \mathrm{ObO}$ the direct limit $S^{1} T A$ of $\left(T^{s}, T^{t s}, s \in \mathfrak{S}(A)\right.$ exists,

(ii) $T$ is S-half exact, and

(iii) $\lim$ is $\left(T, \Im^{\prime}, \delta\right)$-exact.

Then $\left(T, \overrightarrow{S^{1}} T, \delta\right)$ is exact, i.e. for every $a \in \mathfrak{S}$ the sequence

$T Q a \longrightarrow T Z a \longrightarrow T C o k a \stackrel{\delta}{\longrightarrow} S^{1} T Q a \longrightarrow S^{1} T Z a \longrightarrow S^{1} T$ Coka is exact.

Proof. (1) Exactness of the sequence $T Z a \rightarrow T$ Cok $a \rightarrow S^{1} T Q a$. 'For every $s \in \mathfrak{S}(Z a)$ consider the commutative diagram with exact rows

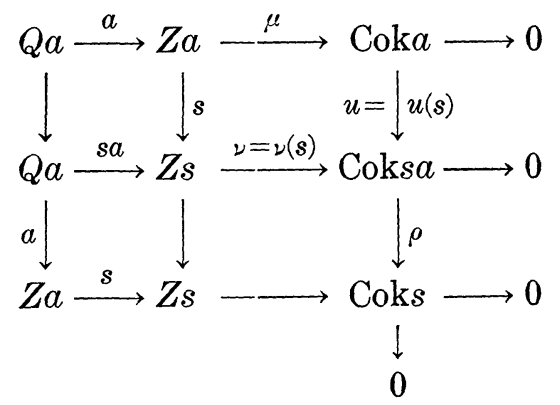

5) We use the word $\left(T, \mathbb{S}^{\prime}, \delta\right)$-exact since the exactness depends on $T$ and $\mathfrak{S}^{\prime}$ and is used for $\delta$-functors. Later on we shall also introduce the notions of $\left(T, \mathbb{S}^{\prime}\right.$, $\varphi)$-resp. $(T, \mathfrak{S}, \rho)$-exactness.

6) The morphisms $u(s)$ and $\nu(s)$ of this diagram obviously depend on $s$. We shall shortly write $u$ resp. $\nu$ instead of $u(s)$ resp. $\nu(s)$ where this cannot lead to errors. 
The last column is exact, and $u(s) \in \mathfrak{S}$ by 2.5. This diagram gives rise to the commutative exact diagram

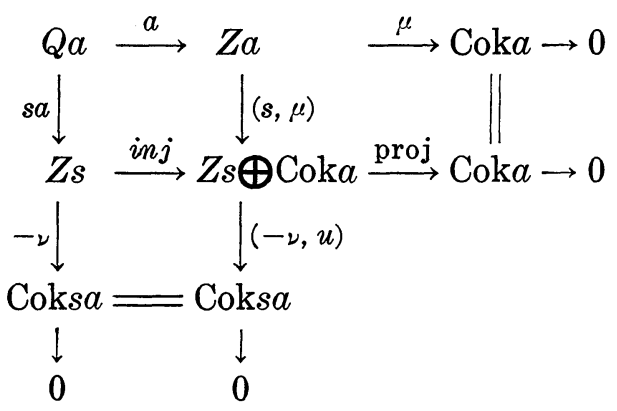

since the upper left square is cocartesian; this in turn implies the commutative exact diagram

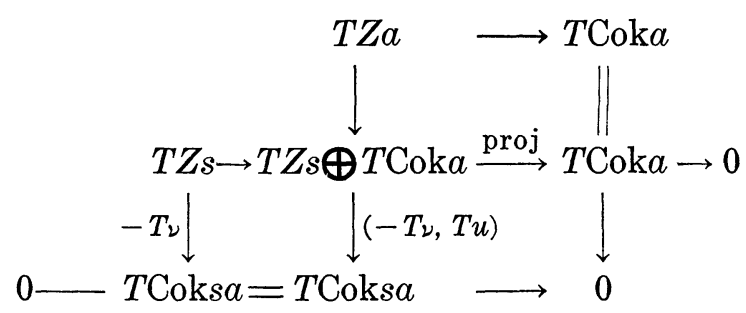

since $T$ is 5 -half exact. By Lemma 3.3, Ch. 3 [2], there results the commutative exact diagram

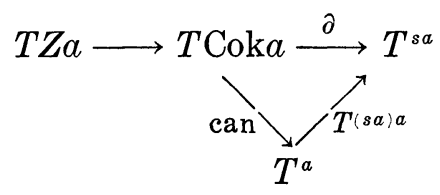

where $\partial$ is the connecting morphism from this lemma. The commutativity is easily verified. Hence $T^{(s a) a}$ is a monomorphism for all $s \in \mathfrak{S}(Z a)$. For every $t \in \mathfrak{S}^{\prime}(Q a)$ let now $a_{t}^{\prime} \in \mathfrak{S}(Z t)$ be uniquely determined by the cocartesian diagram

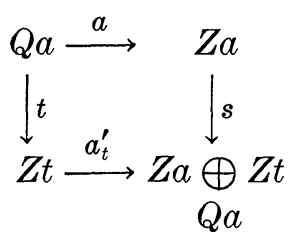

where $s \in \mathfrak{S}$ by (C2). Hence also $T^{a} \longrightarrow T^{a_{t}^{\prime} t}$ is a monomorphism for every $t \in \mathfrak{S}^{\prime}(Q \alpha)$. Moreover the class $\left\{a_{t}^{\prime} t \mid t \in \mathfrak{S}^{\prime}(Q \alpha)\right\}$ is cofinal in $\widetilde{S}^{\prime}(Q \alpha)$ by $\left(\mathrm{C}^{\prime} 1\right)$ and $\left(\mathrm{C}^{\prime} 2\right)$. Hence since $\lim$ is $\left(T, \mathfrak{S}^{\prime}, \delta\right)$-exact we obtain that 


$$
T^{a} \longrightarrow \underset{t}{\lim } T^{a_{t}^{\prime} t}=S^{1} T Q a
$$

is a monomorphism, and thus

$$
T Z a \longrightarrow T \operatorname{Cok} a \stackrel{\delta}{\longrightarrow} S^{1} T Q a \text { is exact . }
$$

Moreover we get at once for every $A$ and $s \leqq t$ in $\mathfrak{S}(A)$ that the morphism $T^{t s}$ is a monomorphism; for the diagram

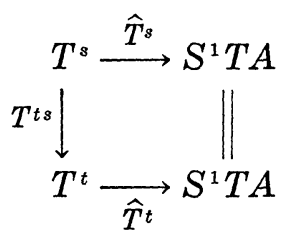

is commutative, and $\hat{T}^{s}$ is a monomorphism by the preceding proof.

(2) Exactness of $S^{1} T Q a \longrightarrow S^{1} T Z a \longrightarrow S^{1} \operatorname{Cok} a$.

The diagram 5.3 from (1) gives rise to the commutative exact diagram

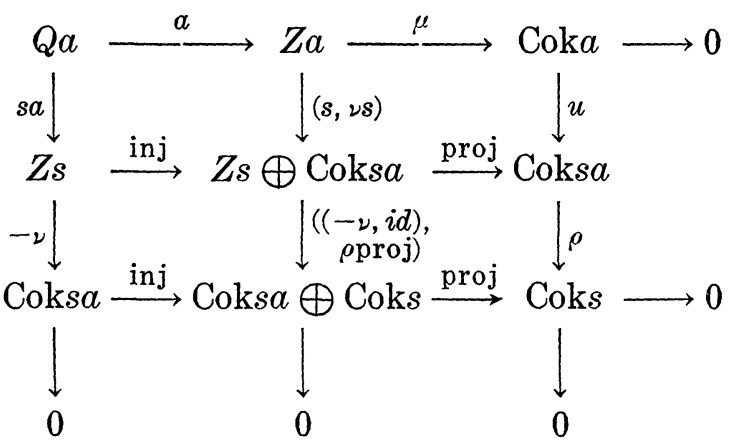

Here also $(s, \nu s) \in \mathfrak{S}$ by $\S 2$. Since all rows are exact, and the bottom row splits, and since the outer columns are exact, also the middle column is exact. This is seen by applying the functors $\mathfrak{A}\left(\right.$ ?,$\left.A^{\prime}\right)$, $A^{\prime} \in 0 b \mathfrak{A}$, to this diagram, and proving the dual result for Abelian groups. Since $T$ is S-half exact, and by Lemma 3.3, Ch. 3 [2], we obtain the commutative exact diagram

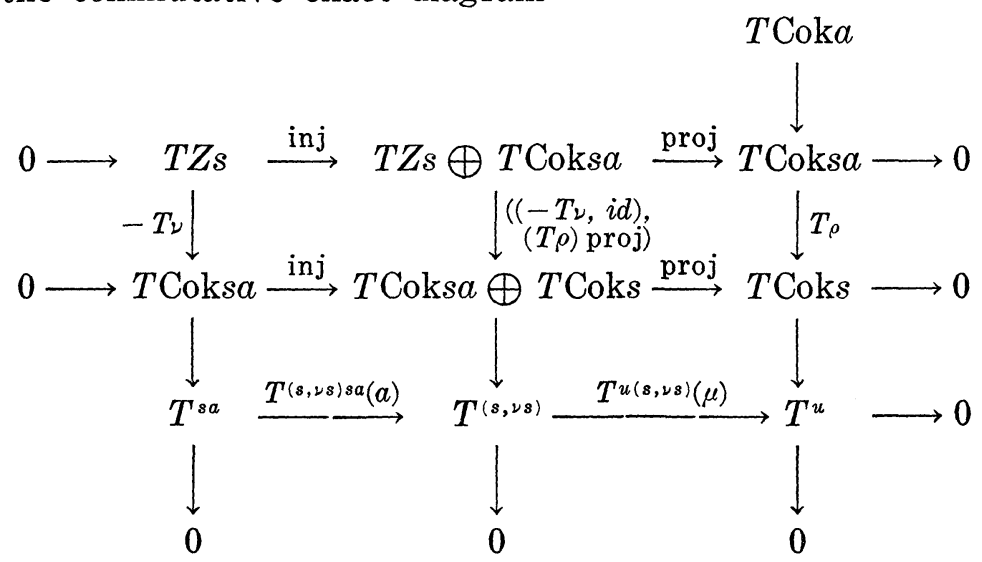


Let now $I$ be the class of all $(r, s, t), r \in \mathbb{S}^{\prime}(Q a), s \in \mathfrak{S}^{\prime}(Z a), t \in \mathfrak{S}^{\prime}(\operatorname{Cok} a)$ with $r \leqq s a$ and $u(s) \leqq t$, preordered componentwise. Since $T^{t u(s)}$ is a monomorphism by 1) we obtain an exact sequence

$$
T^{s a} \longrightarrow T^{(s, \nu(s) s)} \longrightarrow T^{t}
$$

for every $(r, s, t) \in I$. But since $r \leqq s a$ and $s \leqq(s, \nu(s) s)$ the morphisms sa resp. $(s, \nu(s) s)$ are contained in $\widetilde{S}^{\prime}(Q a)$ resp. $\mathfrak{S}^{\prime}(Z a)$ by $\left(\mathrm{C}^{\prime} 2\right)$. Moreover the classes

$$
\{s a \mid(r, s, t) \in I\} \text { resp. }\{(s, \nu(s) s) \mid(r, s, t) \in I\} \text { resp. }\{t \mid(r, s, t) \in I\}
$$

are cofinal in $\mathscr{S}^{\prime}(Q a)$ resp. $\mathfrak{S}^{\prime}(Z a)$ resp. $\mathfrak{S}^{\prime}(\operatorname{Cok} a)$. Hence since $\lim$ is $\left(T, \mathfrak{S}^{\prime}, \delta\right)$-exact we obtain the exact sequence

$$
\stackrel{\lim }{\longrightarrow} T^{s a}=S^{1} T Q a \longrightarrow \lim T^{(s, \nu(s) s)}=S^{1} T Z a \longrightarrow \underline{\lim } T^{t}=S^{1} T \operatorname{Cok} a .
$$

(3) Exactness of the sequence $T$ Cok $a \longrightarrow S^{1} T Q a \longrightarrow S^{1} T Z a$. The commutative diagram 5.4 gives rise, by Lemma 3.3, Ch. 3 [2], to the commutative exact diagram

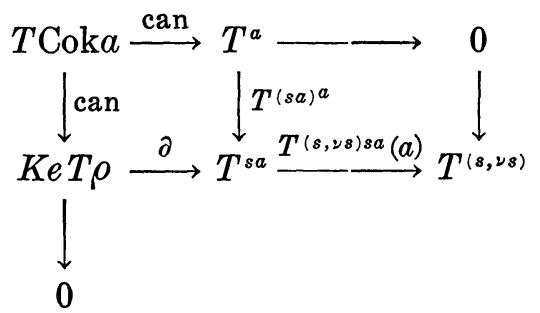

where $\partial$ is the connecting morphism from this lemma. The commutativity is easily checked. Hence if we use $s \leqq(s, \nu s) \leqq s$ we obtain the exact sequence

$$
T^{a} \longrightarrow T^{s a} \longrightarrow T^{s} \text { for every } s \in \mathfrak{S}^{\prime}(Z a) .
$$

Analogous to (2) there results the exact sequences

$$
T^{a} \longrightarrow S^{1} T Q a \longrightarrow S^{1} T Z a,
$$

and then also

$$
T \operatorname{Cok} a \longrightarrow S^{1} T Q a \longrightarrow S^{1} T Z a \text {. }
$$

Similar but easier proofs show the following two theorems.

Let again $T: \mathfrak{A} \longrightarrow \mathfrak{B}$ be an additive functor, and assume that for every $A \in 0 b \mathfrak{A}$ the direct limit of $\left(T_{s}, T_{t s}, s \in \mathfrak{S}(A)\right)$ exists. Then $\lim$ is called $\left(T, \mathfrak{S}^{\prime}, \varphi\right)$-exact if the following condition holds: Given $\overrightarrow{\text { the }}$ data $(a),(b),(c)$ from 5.1 satisfying 
(i) as in 5.1

(ii) $T_{\alpha_{0}(i)} \stackrel{T_{\alpha_{1}(i) \alpha_{0}(i)}\left(a_{1}\right)}{\longrightarrow} T_{\alpha_{1}(i)} \stackrel{T_{\alpha_{2}(i) \alpha_{1}(i)}\left(a_{2}\right)}{\longrightarrow} T_{\alpha_{2}(i)}$ exact for $i \in I$

(iii) the images $\operatorname{Im} \alpha_{\lambda}$ of all three $\alpha_{\lambda}$ are cofinal and contained in $\mathfrak{S}^{\prime}$, or $A_{0}=0,\left|\operatorname{Im} \alpha_{1}\right|=1$ and $\operatorname{Im} \alpha_{2}$ cofinal and contained in $\mathfrak{S}^{\prime}$ then also the sequence

$$
\stackrel{\lim }{\longrightarrow} T_{\alpha_{0}}(i) \longrightarrow \lim _{\alpha_{1}}(i) \longrightarrow \lim T_{\alpha_{2}}(i)
$$

is exact.

THEOREM 5.5. Let $T: \mathfrak{A} \longrightarrow \mathfrak{B}$ be an additive functor, and $\mathfrak{S}^{\prime} a$ subclass of $\mathfrak{S}$ satisfying $\left(\mathrm{C}^{\prime} 1\right)$ and $\left(\mathrm{C}^{\prime} 2\right)$. Assume that

(i) for every $A \in 0 b \mathfrak{A}$ the direct limit $R T A$ of $\left(T_{s}, T_{r s}, s \in \mathfrak{S}(A)\right)$ exists,

(ii) $T$ transforms $\mathfrak{S}$ into Mon $\mathfrak{B}$, and

(iii) $\lim$ is $\left(T, \mathfrak{S}^{\prime}, \varphi\right)$-exact.

Then $R T$ is S-left exact, and $T \stackrel{\tilde{\varphi}}{\longrightarrow} R T$ is a monomorphism, and hence $R T=R^{0} T$ is the 0-th $\subseteq$-derived functor of $T$.

The proof also uses the diagram 5.4 of the proof of Theorem 5.2, and is left to the reader.

Finally if $T: \mathfrak{A} \longrightarrow \mathfrak{B}$ is an additive functor such the direct limit $M T A$ of $\left({ }_{s} T,{ }_{t s} T, s \in \mathfrak{S}(A)\right)$ exists for every $A \in 0 b \mathfrak{A}$ and if $\mathfrak{S}^{\prime}$ is a subclass of $\mathfrak{S}$ satisfying $\left(\mathrm{C}^{\prime} 1\right)$ and $\left(\mathrm{C}^{\prime} 2\right)$ we call $\lim \left(T, \mathfrak{S}^{\prime}, \rho\right)$-exact if the following condition holds:

Given a morphism $a: A_{0} \longrightarrow A_{1}$, a filtered class $I$ and order preserving maps $\alpha_{\lambda}: I \longrightarrow \mathfrak{S}^{\prime}\left(A_{\lambda}\right), \lambda=0,1$, with cofinal image satifying

(i ) $\alpha_{0}(i) \leqq \alpha_{1}(i) a$ for $i \in I$

(ii) $\alpha_{0}(i) T \stackrel{\alpha_{1}(i) \alpha_{0}(i)}{\longrightarrow} \underset{\alpha_{1}(i)}{\longrightarrow} T$ monomorphism then also $\lim _{\alpha_{0}(i)} T \longrightarrow \lim _{\alpha_{1}(i)} T$ is a monomorphism.

THEOREM 5.6. Let $T: \mathfrak{A} \longrightarrow \mathfrak{B}$ be an additive functor, and $\mathfrak{S}^{\prime}$ a subclass of satisfying $\left(\mathrm{C}^{\prime} 1\right)$ and $\left(\mathrm{C}^{\prime} 2\right)$. Assume that that

(i) for every $A \in 0 b \mathfrak{A} M T A=\lim \left({ }_{s} T,{ }_{t s} T, s \in \mathfrak{S}(A)\right)$ exists, and

(ii) $\stackrel{\lim }{\longrightarrow}$ is $\left(T, \mathcal{S}^{\prime}, \rho\right)$-exact.

Then $M T$ transforms $\subseteq$ into Mon $\mathfrak{B}$, and hence $M^{\circ} T=M T$.

6. Application to special cases. We apply the general theorems of the paragraphs 4 and 5 to the three special cases mentioned in the introduction. 
Case (I). Let $T: \mathfrak{A} \longrightarrow \mathfrak{B}$ be an additive functor.

Definition 6.1 (see [9]). An object $N$ in $\mathfrak{A}$ is $\mathfrak{S}$-T-injective if and only if for all $s \in \mathfrak{S}(N)$ the sequence $T N \longrightarrow T Z s \longrightarrow T$ Coks $\longrightarrow 0$ is exact.

Proposition 6.2 ([9]). The class of $\mathfrak{S}$ - $T$-injective objects in $\mathfrak{A}$ is closed under finite direct sums.

Let then $\mathfrak{S}^{\prime}$ be the class of all $t \in \mathfrak{S}$ such that there is an $s \in \mathbb{S}$ with $\subseteq$ - $T$-injective $Z s$ and $s \leqq t$. Obviously $\mathfrak{S}^{\prime}$ satisfies condition $\left(\mathrm{C}^{\prime} 2\right)$.

Proposition 6.3. Assume that $\mathfrak{A}$ has enough $\mathfrak{S}$ - $T$-injective objects, i.e. for every $A \in 0 \mathrm{~b} \mathfrak{A}$ there is an $s \in \mathfrak{S}(A)$ with $\subseteq$ - $T$-injective codomain.

(i) (a) For every $A \in 0 \mathrm{~b} \mathfrak{A}$ the class $\mathfrak{S}^{\prime}(A)$ is cofinal in $\mathfrak{S}(A)$, i.e. $\mathfrak{S}^{\prime}$ satisfies $\left(\mathrm{C}^{\prime} 1\right)$.

(b) For every $A \in 0 \mathrm{~b} \mathfrak{A}$ and $s \leqq t$ in $\mathfrak{S}^{\prime}(A)$ the morphism $T^{t s}$ is an isomorphism. Hence $\stackrel{\lim }{\longrightarrow}\left(T^{s}, T^{t s}, s \in \subseteq(A)\right)$ exists, and $\underset{\lim }{\longrightarrow}$ is $\left(T, \mathfrak{S}^{\prime}, \delta\right)$-exact.

(ii) Assume that $T$ transforms $\subseteq$ into Mon $\mathfrak{B}$. Then for every $A \in 0 \mathrm{bA}$ and $s \leqq t$ in $\mathfrak{S}^{\prime}(A)$ the morphism $T_{t s}$ is an isomorphism. Hence $\stackrel{\lim }{\longrightarrow}\left(T_{s}, T_{t s}, s \in \mathfrak{S}(A)\right)$ exists, and the functor $\stackrel{\lim }{\longrightarrow}$ is $\left(T, \mathbb{S}^{\prime}, \varnothing\right)$ exact.

As an immediate consequence of the preceding proposition and the Theorems 4.3, 4.9, 5.2, 5.5 we obtain the:

Theorem 6.4. Assume that $\mathfrak{A}$ has enough $\mathfrak{S}$-T-injectives. Then

(i) the first satellite $S^{1} T$ of $T$ exists. If $T$ is S-half exact, then $\left(T, S^{1} T, \delta\right)$ is exact.

(ii) If $T$ tranforms $\subseteq$ into Mon $\mathfrak{B}$, then the 0-th derived functor $R^{\circ} T$ of $T$ exists, and the morphism $\tilde{\varphi}: T \longrightarrow R^{\circ} T$ is a monomorphism.

Proof of the proposition. (i) (a) For every $s \in \mathbb{S}$ there is a $t \in \mathfrak{S}(Z s)$ with $\mathfrak{S}$ - $T$-injective $Z t$. But then $s \leqq t s$ and $t s \in \mathfrak{S}^{\prime}(Q s)$.

(b) Let $s, t \in \subseteq(A)$. We obtain the commutative exact diagram

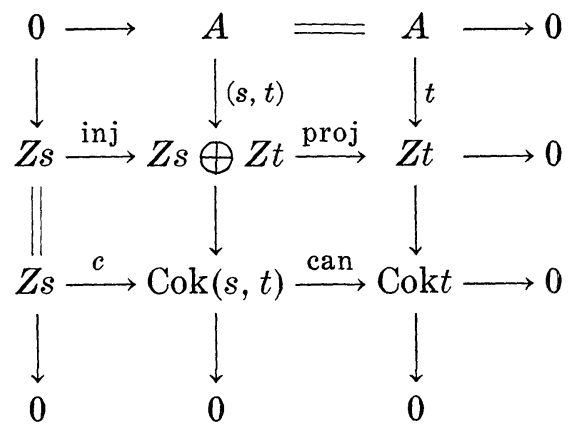


where $c \in \mathfrak{S}$ by 2.6. If now $Z s$ is $\mathfrak{S}-T$-injective then we obtain the commutative exact diagram

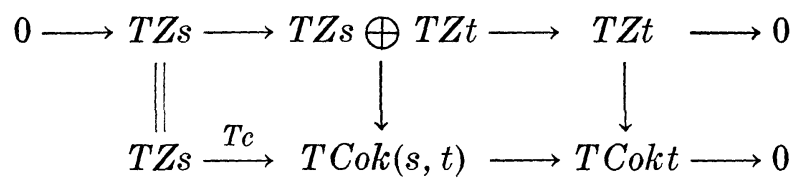

Lemma 3.2, Ch. 3 [2], at once implies that $T^{t(s, t)}$ is an isomorphism. If moreover $s \leqq t$, then $s \leqq(s, t) \leqq s$, and thus $T^{(s, t) s}$ is an isomorphism. So $T^{t s}$ is isomorphic for $s \leqq t$ and $\subseteq$ - $T$-injective $Z s$. By definition of $\mathfrak{S}^{\prime}$ this result then also holds for all $s \leqq t, s, t \in \mathfrak{S}^{\prime}$.

(ii) If $T$ transforms $\subseteq$ into Mon $\mathfrak{B}$ then $T c$ is a monomorphism. Lemma 3.3, Ch. 3 [2], applied to the diagram 6.5 immediatly shows that $T_{t(s, t)}$ is an isomorphism. The remainder is shown as in (i).

CoRollary 6.6. Hypotheses as in 6.4. If $N$ is S-T-injective then $S^{1} T N=0$. If $T$ is 5 -half exact and $S^{1} T A=0$ for an object $A \in 0 \mathrm{~b} \mathfrak{A}$ then $A$ is $\subseteq$-T-injective.

REMARK 6.7. If $\mathfrak{A}$ is Abelian and $\subseteq$ the class of all monomorphisms of $\mathfrak{X}$ then Theorem 6.4, (i) improves the corresponding results of H. Röhrl in [9]. On the other side the proof given here uses the ideas of [9].

We finally show the existence of all satellites and derived functors if the following condition is satisfied (see also [9]).

(C) If $s \in \mathfrak{S}$ and $Q s$ and $Z s$ are $\mathfrak{S}$-T-injective then also Coks is ธ-T-injective.

THEOREM 6.8. Assume that there are enough $\mathfrak{S}$-T-injectives and that condition (C) is satisfied. Then

(i) every $\widetilde{S}$-T-injective object is also $\widetilde{S}-S^{1} T$-injective. Hence, by induction, all satellites $S^{n} T$ of $T$ exist. If moreover $T$ is $\subseteq$-half exact the family $\left(S^{n} T, \delta^{n}\right)$ is an exact $\subseteq$ - $\delta$-functor.

(ii) if $T$ transforms $\subseteq$ into Mon $\mathfrak{B}$ every $\mathfrak{S}$-T-injective object is also $\subseteq$ - $R^{\circ} T$-injective. Hence all derived functors $R^{n} T$ of $T$ exist, and $\left(R^{n} T, \delta^{n}\right)$ is exact. Moreover there is a unique morphism $\left(S^{n} T, \delta^{n}\right) \longrightarrow\left(R^{n} T, \delta^{n}\right)$ extending $\tilde{\varphi}: T \longrightarrow R^{\circ} T$.

Proof. Let $N \stackrel{a}{\longrightarrow} Z a \longrightarrow C o k a \longrightarrow 0$ be exact with $a \in \subseteq$ and $\mathfrak{S}$ - $T$-injective $N$. Let $s \in \mathfrak{S}(Z a)$ with $\mathfrak{S}$-T-injective $Z s$. We obtain the commutative exact diagram 


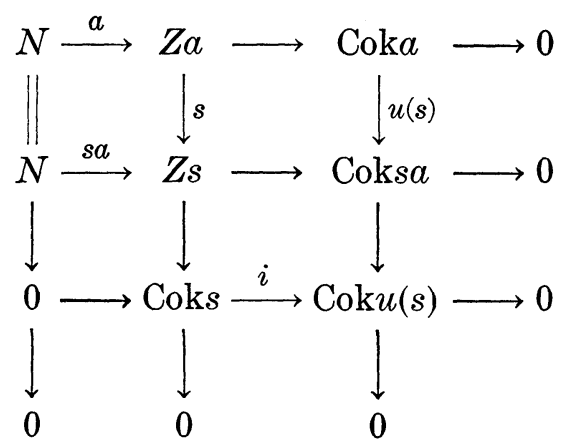

where Coksa is $\widetilde{S}$-T-injective by (C) and $i$ is an isomorphism. This diagram gives rise to the commutative exact diagram

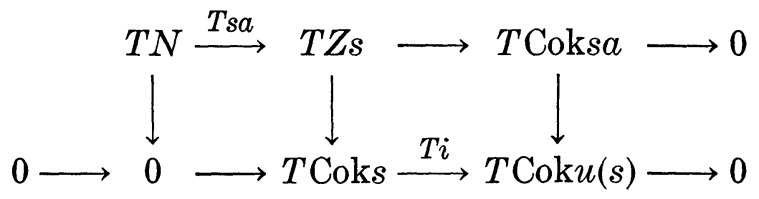

which in turn by Lemma 3.3, Ch. 3 [2], implies the exact sequence

$$
T N \longrightarrow T_{s} \longrightarrow T_{u(s)} \longrightarrow 0 \longrightarrow T^{s} \longrightarrow T^{u(s)} \longrightarrow 0 \text {. }
$$

Hence we obtain the exact sequence

$$
S^{1} T N=0 \longrightarrow S^{1} T Z a \longrightarrow S^{1} T \operatorname{Cok} a \longrightarrow 0,
$$

and also the exact sequence

$$
T N=R^{\circ} T N \longrightarrow R^{\circ} T Z a \longrightarrow R^{\circ} T \operatorname{Cok} a \longrightarrow 0
$$

if $T$ transforms $\subseteq$ into Mon $\mathfrak{B}$. So $N$ is $\subseteq$ - $S^{1} T$-injective, and also $\Im$ - $R^{\circ} T$-injective under the additional hypothesis.

CoRollary 6.9. Hypothesis as in 6.8, (ii). Then one can calculate the $R^{n} T A$ for every $A \in 0 \mathrm{bO}$ in the following usual manner: Let

$$
0 \longrightarrow A \longrightarrow I^{0} \stackrel{d^{0}}{\longrightarrow} I^{1} \stackrel{d^{1}}{\longrightarrow} I^{2} \longrightarrow \cdots
$$

be an $\mathfrak{S}$-T-injective resolution of $A$, i.e. an $\mathfrak{S}$-exact sequence with ธ-T-injective objects $I^{n}$. Then (setting $d^{-1}:=0$ )

$$
R^{n} T A=\operatorname{Ke} T d^{n} / \operatorname{Im} T d^{n-1} \text { for } n \geqq 0 \text {. }
$$

REMARK 6.10. If $T$ is any additive functor then $M^{0} T$ exists if the category $\mathfrak{B}$ has exact filtered direct limits, and if the classes of subobjects are sets (see proposition 4.7). 
Case (II). Assume that for every $A \in 0 \mathrm{~b} \mathfrak{A}$ the class $\subseteq(A)$ has a largest element. Then define $\widetilde{S}^{\prime}$ such that for all $A \in 0 \mathrm{~b} \mathfrak{A} \widetilde{S}^{\prime}(A)$ is the class of all largest elements in $\subseteq(A)$. Obviously $\varsigma^{\prime}$ satisfies the conditions $\left(\mathrm{C}^{\prime} 1\right)$ and $\left(\mathrm{C}^{\prime} 2\right)$, and for every $A$ and $s \leqq t$ in $\widetilde{S}^{\prime}(A)$ the morphisms ${ }_{t s} T, T_{t s}$, and $T^{t s}$ are isomorphisms for an arbitrary functor $T$. Especially, for every $A \in 0 b \mathfrak{A}$, the direct limits $M T A, R T A$, and $S^{1} T A$ exist, and $\lim$ is $\left(T, \mathcal{S}^{\prime}, \rho\right)-,\left(T, \mathcal{S}^{\prime}, \varphi\right)$-, and $\left(T, \Im^{\prime}, \delta\right)$-exact. Hence we obtain the

THEOREM 6.11. Assume that for every $A \in 0 \mathrm{bP}$ the class $\subseteq(A)$ has a largest element.

(i) For any additive functor $T: \mathfrak{A} \longrightarrow \mathfrak{B}$ the functors $M^{0} T$, $S^{n} T, R^{n} T$ for $n \geqq 0$ exist.

(ii) The universal $\mathfrak{S}_{-}-\delta$-functor $\left(R^{n} T, \delta^{n}\right)$ is exact.

(iii) If $T$ is S-half exact then the universal $\widetilde{S}-\delta$-functor $\left(S^{n} T, \delta^{n}\right)$ is exact.

Case (III). Assume that $\mathfrak{A}$ has enough $\subseteq$-injective objects. In this case we can apply Theorem 6.11 since if $A \in 0 b \mathfrak{U}$ and $s \in \mathfrak{S}(A)$ with $\mathfrak{S}$-injective $Z s$ then $s$ is a largest element of $\mathfrak{S}(A)$. The derived functors of any additive functor $T: \mathfrak{A} \longrightarrow \mathfrak{B}$ can again be obtained as the homology objects of the complex

$$
T I^{0} \longrightarrow T I^{1} \longrightarrow T I^{2} \longrightarrow \cdots
$$

where

$$
0 \longrightarrow A \longrightarrow I^{0} \longrightarrow I^{1} \longrightarrow I^{2} \longrightarrow \cdots
$$

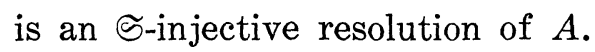

We remark that under the preceding assumptions the closure $\bar{\subseteq}$ of $\mathfrak{S}$ in the sense of [7] is the class morphisms of an injective structure, and that the satellites and derived functors of a functor $T$ with respect to $\widetilde{S}$ are the same as those with respect to $\widetilde{\mathfrak{S}}$. Hence without loss of generality we may assume that $\subseteq$ itself is already an injective structure. Hence one recovers the results of [8] on the existence and exactness of satellites and derived functors with respect to injective structures.

REMARK 6.12. Assume that $T: \mathfrak{A} \longrightarrow \mathfrak{B}$ is $\mathfrak{S}$-left exact. Then for any short $\mathfrak{S}$-exact sequence

$$
0 \longrightarrow A^{\prime} \stackrel{a}{\longrightarrow} A \stackrel{\mu}{\longrightarrow} A^{\prime \prime} \longrightarrow 0
$$

the morphism Cok $\mu \stackrel{0}{\longrightarrow} 0$ is in $\subseteq$. This implies that under the 
hypothesis of 6.4 or 6.11 we have $T \operatorname{Cok} \mu=0$ and $R^{1} T \operatorname{Cok} \mu=0$. Since we also have the exact sequence

$$
\mathrm{Cok} a \stackrel{s}{\longrightarrow} A^{\prime \prime} \stackrel{\text { can }}{\longrightarrow} \mathrm{Cok} \mu=\mathrm{Cok} s \longrightarrow 0
$$

with $s \in \mathbb{S}$ we obtain the long exact sequence

$$
\begin{aligned}
0 \longrightarrow T \operatorname{Cok} a \stackrel{T s}{\longrightarrow} T A^{\prime \prime} \longrightarrow T \operatorname{Cok} \mu=0 \longrightarrow \\
\longrightarrow R^{1} T \operatorname{Cok} a \stackrel{R^{1} T s}{\longrightarrow} R^{1} T A^{\prime \prime} \longrightarrow R^{1} T \operatorname{Cok} \mu=0 .
\end{aligned}
$$

Hence $T s$ and $R^{1} T s$ are isomorphisms, and we also obtain the long exact sequence

$$
0 \longrightarrow T A^{\prime} \stackrel{T a}{\longrightarrow} T A \stackrel{T \mu}{\longrightarrow} T A^{\prime \prime} \stackrel{\delta}{\longrightarrow} R^{1} T A^{\prime} \stackrel{R^{1} T a}{\longrightarrow} R^{1} T a \stackrel{R^{1} T \mu}{\longrightarrow} R^{1} T A^{\prime \prime} .
$$

There results that it was no loss of generality that in the definition of derived functors we restricted ourselves to short $\mathfrak{S}$-exact sequences of the form

$$
0 \longrightarrow Q s \stackrel{s}{\longrightarrow} Z s \stackrel{\text { can }}{\longrightarrow} \text { Coks } \longrightarrow 0 \text {. }
$$

The author thanks the referee for his valuable criticism.

\section{REFERENCES}

1. D. Buchsbaum, Satellites and universal functors, Ann. of Math. 17 (1960).

2. H. Cartan and S. Eilenberg, Homological Algebra, Princeton University Press, 1956.

3. S. Eilenberg and J. C. Moore, Foundations of relative homological algebra, Mem. of the Amer. Math. Soc. 55 (1965).

4. P. Gabriel, Des Catégories Abéliennes, Bull. Soc. Math. (3) 90 Paris, 1961.

5. A. Grothendieck, Sur Quelques Points d'Algèbre Homologique, Tohoku Math. J. 9 (1957).

6. A Grothendieck and J. Dieudonne, Elements De Géometrie Algébrique I; I.H.E.S. Publication Math. 4 (1960).

7. J. M. Maranda, Injective structures, Trans. Amer. Math. Soc. 110 (1964).

8. U. Oberst, Systeme direkt verbundener Kategorien und universelle Funktoren, Dissertation, Universität München, 1964.

9. H. Röhrl, Satelliten halbexakter Funktoren, Math. Zeitschrift 79 (1962).

Received January 2, 1966, and in revised form July 2, 1966. This paper was written with support of NSF grant GP-5870. 


\section{PACIFIC JOURNAL OF MATHEMATICS}

\section{EDITORS}

\section{H. SAMELSON}

Stanford University

Stanford, California

\author{
J. P. JANS \\ University of Washington \\ Seattle, Washington 98105
}

\section{J. DuGUNDJI}

University of Southern California

Los Angeles, California 90007

RichaRd ARENS

University of California

Los Angeles, California 90024

\section{ASSOCIATE EDITORS}

E. F. BECKENBACH
B. H. NeUManN

F. WOLF

K. YOSIDA

\section{SUPPORTING INSTITUTIONS}

UNIVERSITY OF BRITISH COLUMBIA

CALIFORNIA INSTITUTE OF TECHNOLOGY

UNIVERSITY OF CALIFORNIA

MONTANA STATE UNIVERSITY

UNIVERSITY OF NEVADA

NEW MEXICO STATE UNIVERSITY

OREGON STATE UNIVERSITY

UNIVERSITY OF OREGON

OSAKA UNIVERSITY

UNIVERSITY OF SOUTHERN CALIFORNIA
STANFORD UNIVERSITY

UNIVERSITY OF TOKYO

UNIVERSITY OF UTAH

WASHINGTON STATE UNIVERSITY

UNIVERSITY OF WASHINGTON

AMERICAN MATHEMATICAL SOCIETY

CHEVRON RESEARCH CORPORATION

TRW SYSTEMS

NAVAL ORDNANCE TEST STATION

Mathematical papers intended for publication in the Pacific Journal of Mathematics should be typewritten (double spaced). The first paragraph or two must be capable of being used separately as a synopsis of the entire paper. It should not contain references to the bibliography. Manuscripts may be sent to any one of the four editors. All other communications to the editors should be addressed to the managing editor, Richard Arens at the University of California, Los Angeles, California 90024.

50 reprints per author of each article are furnished free of charge; additional copies may be obtained at cost in multiples of 50 .

The Pacific Journal of Mathematics is published monthly. Effective with Volume 16 the price per volume ( 3 numbers) is $\$ 8.00$; single issues, $\$ 3.00$. Special price for current issues to individual faculty members of supporting institutions and to individual members of the American Mathematical Society: $\$ 4.00$ per volume; single issues $\$ 1.50$. Back numbers are available.

Subscriptions, orders for back numbers, and changes of address should be sent to Pacific Journal of Mathematics, 103 Highland Boulevard, Berkeley 8, California.

Printed at Kokusai Bunken Insatsusha (International Academic Printing Co., Ltd.), No. 6, 2-chome, Fujimi-cho, Chiyoda-ku, Tokyo, Japan.

PUBLISHED BY PACIFIC JOURNAL OF MATHEMATICS, A NON-PROFIT CORPORATION

The Supporting Institutions listed above contribute to the cost of publication of this Journal, but they are not owners or publishers and have no responsibility for its content or policies. 


\section{Pacific Journal of Mathematics}

\section{Vol. 21, No. $3 \quad$ BadMonth, 1967}

Richard Allen Askey, A transplantation theorem for Jacobi coefficients . . . 393

Raymond Balbes, Projective and injective distributive lattices .......... 405

Raymond Balbes and Alfred Horn, Order sums of distributive lattices . . . . 421

Donald Charles Benson, Nonconstant locally recurrent functions ........ 437

Allen Richard Bernstein, Invariant subspaces of polynomially compact operators on Banach space ............................... 445

Robert F. Brown, Fixed points and fibre ................... 465

David Geoffrey Cantor, On the Stone-Weierstrass approximation theorem for valued fields ................................ 473

James Walton England, Stability in topological dynamics .............. 479

Alessandro Figà-Talamanca and Daniel Rider, A theorem on random

Fourier series on noncommutative groups.................. 487

Sav Roman Harasymiv, A note of dilations in $L^{p} \ldots \ldots \ldots \ldots \ldots \ldots . \ldots 493$

J. G. Kalbfleisch, A uniqueness theorem for edge-chromatic graphs ....... 503

Richard Paul Kelisky and Theodore Joseph Rivlin, Iterates of Bernstein polynomials .................................... 511

D. G. Larman, On the union of two starshaped sets ............... 521

Henry B. Mann, Josephine Mitchell and Lowell Schoenfeld, Properties of differential forms in $n$ real variables ...................... 525

John W. Moon and Leo Moser, Generating oriented graphs by means of team comparisons .

Veikko Nevanlinna, A refinement of Selberg's asymptotic equation ...

Ulrich Oberst, Relative satellites and derived functors of functors with additive domain ..............................

John Vincent Ryff, On Muirhead's theorem...............

Carroll O. Wilde and Klaus G. Witz, Invariant means and the Stone-Čech compactification 